\title{
RÉCHAUFFEMENT GLOBAL ET SANTÉ : ASPECTS GENERAUX ET QUELQUES PARTICULARITES DU MONDE TROPICAL
}

\author{
F. MENDONÇA \\ Universidade Federal do Paraná, Curitiba, Brésil \\ chico@ufpr.br
}

\section{Résumé}

Les changements climatiques des deux derniers siècles constituent, aujourd'hui, un fait incontestable. Une élévation de l'ordre de 3 à $6^{\circ} \mathrm{C}$ de la température moyenne de la troposphère dans les prochains cent ans constitue une prévision que l'on accepte de manière générale. Les répercussions positives et négatives de ce réchauffement sur la nature et sur la société appartiennent encore au domaine de la spéculation, mais les répercussions négatives sont assez inquiétantes. L'incidence de certaines maladies aura tendance à diminuer mais celle de beaucoup d'autres, principalement des maladies transmissibles et infectieuses (paludisme, dengue, leptospirose, etc.) dans les tropiques, aura tendance à augmenter dans des conditions climatiques plus chaudes. L'expansion des régions les plus réchauffées vers des latitudes et vers des altitudes plus élevées sera accompagnée de l'expansion géographique de ces maladies.

Mots-clés : Changements globaux, réchauffement troposphérique, maladies, Tropiques.

\begin{abstract}
The global climate change of the two past centuries is today an undeniable fact. A rise of about $3^{\circ}$ to $6^{\circ} \mathrm{C}$ of the average temperature of the troposphere in the next hundred years is worldly accepted. The positive and negative effects of global warming upon nature and society are still speculative, but the negative ones are rather alarming. The occurrence of some diseases will tend to decrease, but that of many others, especially of the transmissible and infectious ones (dengue fever, malaria, etc.) in the tropics will tend to rise in warmer climatic conditions. The expansion of warmer areas to higher latitudes and altitudes will be followed by the expansion of the geographic extent of these diseases.
\end{abstract}

Keywords : climate change, warming of the troposphere, diseases, Tropics.

\section{Resumo}

As mudanças climáticas dos dois últimos séculos constituem, hoje, um fato incontestável. Uma elevação da ordem de $3^{\circ}$ a $6^{\circ} \mathrm{C}$ na temperatura média da troposfera nos próximos cem anos constitui uma previsão aceita de maneira geral. As repercussões positivas e negativas deste aquecimento sobre a natureza e a sociedade são, sobretudo, ainda especulativas, mas as segundas são bastante preocupantes. A incidência de 
algumas enfermidades tenderá a diminuir mas, de muitas, principalmente as transmissíveis e infecciosas (malária, dengue, leptospirose, etc.) nos tropicos, tenderá a se agravar em condições de maior calor. A expansão das áreas mais aquecidas para latitudes e altitudes mais elevadas far-se-á acompanhar pela expansão da área geográfica destas doenças.

Palavras Chave : Mudanças globais, aquecimento troposférico, doenças, tropico.

"While global estimates of climate change are framed by uncertainty, and forecasts of place-to-place variations are also error-prone, we can speculate about, and also consider recent research evidence on the possible or problable health consequences."

Antony Gatrel (2002, p.243-244)

\section{Introduction}

Sur l'élévation des températures de l'atmosphère terrestre, particulièrement de la troposphère, génériquement appelée réchauffement global, planent encore bien des doutes en ce qui concerne ses causes et ses conséquences, même si les données les plus récentes ont prouvé l'intensification du réchauffement de la planète. C'est cette condition qui explique que le réchauffement global constitue une des principales questions de la société d'aujourd'hui en face aussi bien de l'enjeu que représente la connaissance approfondie du réchauffement que des répercussions possibles qu'il a sur la vie des hommes dans la société présente et future.

Du fait qu'il s'agit d'une problématique qui implique, simultanément, la dimension naturelle de la planète et la société humaine qui l'habite, il faut réfléchir à la question du réchauffement planétaire dans une perspective interdisciplinaire. C'est donc, dans ce contexte, que la climatologie, particulièrement son approche géographique, révèle d'amples possibilités en faveur d'un traitement englobant de cette thématique, car elle permet de se rapprocher de la perspective naturelle et de la perspective sociale de la problématique et de l'intégrer à son étude ; c'est en accord avec cette sous branche de la connaissance scientifique que le présent texte a été élaboré.

L’approche qui y est développée met en évidence la réflexion sur la manière dont les changements climatiques globaux peuvent se répercuter sur les conditions de santé de la population, c'est-à-dire :

- Quels genres de complications affectant la santé humaine découleraient de conditions climatiques plus chaudes que les conditions actuelles?

- Quels genres de maladies subiraient une intensification et quels genres de maladies seraient défavorisés ?

- En reviendrait-on à des conceptions telles que le déterminisme environnemental, ou bien les progrès techniques de la société moderne 
et contemporaine, élimineraient la sensibilité humaine aux changements climatiques globaux et régionaux?

Ces questions et bien d'autres encore proposent des enjeux considérables aux experts qui étudient l'interaction entre le climat et la société à l'heure actuelle. $\mathrm{Vu}$ qu'il s'agit d'une réflexion sur une situation future, l'analyse développée dans ce texte focalise sur une discussion au sujet des répercussions possibles d'un réchauffement planétaire présenté sous la forme d’une hypothèse basée sur les prémisses du présent ; la certitude que l'avenir, dans son déroulement, nous réserve de nombreuses surprises confère un caractère spéculatif à cette approche de la question, qui n’est pas distante de la réalité pour autant.

L’épigraphe présentée ci-dessus illustre la perspective qu’on se propose d'esquisser: le climat, en tant qu'élément de l'environnement, exerce une influence considérable sur les conditions de santé et de maladie de la population, mais il ne les détermine pas de façon isolée (Sorre, 1984) ; il subsiste encore tant de questions et de doutes quant au réchauffement global actuel qu'une analyse critique de ses conséquences sur l'état de santé des individus et de la société s’impose (Gatrel, 2002).

La discussion élaborée dans ce texte se situe, de manière générale, dans l'ensemble des discussions relatives à la géographie médicale et de la santé, un champ d'études de la géographie qui reprend de la vigueur et de l’importance de nos jours au Brésil et dans certains pays occidentaux, d’après la conception qu'en ont Iniguez Rojas (1998), Mendonça (2000) et Czeresnia et Ribeiro (2000).

\section{Réchauffement global : aspects généraux}

L'histoire naturelle met en évidence que l'évolution des conditions thermiques de la surface de la terre ne s'est pas produite selon un processus uniforme. Des périodes plus chaudes alternent avec des périodes moins chaudes tout au long de l'histoire naturelle et humaine de la planète.

L'une des caractéristiques de l'atmosphère terrestre, c'est sa capacité à laisser filtrer le rayonnement solaire de courtes longueurs d'ondes et "piéger" le rayonnement infrarouge tellurique, mécanisme qui est connu sous le nom d'effet de serre et qui a son origine dans la dynamique naturelle de la planète même. Le phénomène qui, de nos jours, inquiète tellement la société est l'intensification du réchauffement de la basse atmosphère, en particulier, de la troposphère, la couche qui fait l'objet des études de la climatologie. Tout en ayant une origine naturelle, le réchauffement observé à l'époque actuelle, traité au sein de toutes les discussions sur les changements globaux, semble être directement lié aux activités humaines ; c’est la constatation qui résulte de la plupart des études consacrées à l'évolution de la température de l'atmosphère terrestre. 
D’après McGregor et Nieuwolt (1998, p. 311), les «changements climatiques peuvent être considérés à deux échelles temporelles: des changements de longue durée qui sont supérieurs à 20.000 ans et des changements de courte durée qui peuvent se produire entre 100 et 20.000 ans. La variabilité climatique se réfère à des changements qui ont lieu de décennie en décennie et d'année en année ». Selon ces chercheurs, les origines de ces changements climatiques seraient liées à des causes externes, à des facteurs internes et aux activités humaines (tableau 1).

\begin{tabular}{|c|c|c|}
\hline CAUSES EXTERNES & FACTEURS INTERNES & ACTIVITÉS HUMAINES \\
\hline $\begin{array}{l}\text { Changements affectant } \\
\text { l'orbite de la planète } \\
\text { Variation du rayonnement } \\
\text { solaire. }\end{array}$ & $\begin{array}{l}\text { Changements affectant la } \\
\text { circulation océanique } \\
\text { Changements affectant la } \\
\text { composition des gaz de } \\
\text { l'atmosphère } \\
\text { (principalement CO2, CH4 } \\
\text { et O3) } \\
\text { Changements affectant les } \\
\text { conditions de la couche } \\
\text { géographique. }\end{array}$ & $\begin{array}{l}\text { Combustion de } \\
\text { combustibles fossiles. } \\
\text { Envoi de gaz à effet de } \\
\text { serre dans l'atmosphère. } \\
\text { Déforestation. } \\
\text { Modification climatique } \\
\text { aux échelles régionale et } \\
\text { locale. }\end{array}$ \\
\hline
\end{tabular}

Tableau 1 : Causes des changements climatiques. Source: McGregor et Nieuwolt, 1998. Organisation : F. Mendonça.

Les causes externes sont liées à des changements affectant l'orbite de la planète autour du soleil et elles se répercutent directement sur la variation du rayonnement solaire. Les facteurs internes sont représentés par des changements observés dans les océans, dans l'air et dans le relief et se rattachent à des mécanismes de feed-back non linéaires ou à des interactions des composants du système climatique; n'importe quel changement qui se produira dans l'un d'entre eux se répercutera sur les autres. Des changements affectant la circulation océanique sont liés à des altérations de la température, de la salinité et du mouvement des courants marins; des changements affectant la composition des gaz atmosphériques peuvent indiquer l'élévation ou la diminution de la quantité des gaz à effet de serre (GES), par exemple, de ceux dont les sources et l'absorption sont amplement connues (tableau 2); enfin, les changements affectant la couche géographique se réfèrent au mouvement de plaques tectoniques, à l'isostasie continents-océans, à l'activité volcanique, etc., tout cela pouvant se refléter en altérations dans le bilan d'énergie soleil-terreespace.

\begin{tabular}{|c|l|l|}
\hline Gaz & Source & Absorption \\
\hline CO2 & $\begin{array}{l}\text { Combustibles fossiles, déforestation, } \\
\text { combustion de biomasse, production de } \\
\text { ciment. }\end{array}$ & $\begin{array}{l}\text { Océan et biosphère } \\
\text { terrestre. }\end{array}$ \\
\hline
\end{tabular}




\begin{tabular}{|c|l|l|}
\hline CH4 & $\begin{array}{l}\text { Plantations de riz, marécages naturels, } \\
\text { animaux domestiques ruminants, } \\
\text { combustion de biomasse, combustibles } \\
\text { fossiles, termites, déchets domestiques et } \\
\text { animaux. }\end{array}$ & $\begin{array}{l}\text { Réaction avec des } \\
\text { radicaux hydroxydes dans } \\
\text { l'atmosphère. }\end{array}$ \\
\hline N2O & $\begin{array}{l}\text { Sources biologiques dans le sol et dans } \\
\text { l'eau, fertilisation à base d'engrais, } \\
\text { combustion de biomasse et industrie. }\end{array}$ & $\begin{array}{l}\text { Destruction photolytique } \\
\text { dans la stratosphère. }\end{array}$ \\
\hline Halo carbures & $\begin{array}{l}\text { Sources industrielles : sprays, } \\
\text { réfrigérants, dissolvants, extincteurs } \\
\text { d'incendie, agents producteurs d'écume. }\end{array}$ & $\begin{array}{l}\text { Destruction photolytique } \\
\text { dans la stratosphère. }\end{array}$ \\
\hline H2O & $\begin{array}{l}\text { Évaporation (océan), traînée de } \\
\text { condensation (trafic aérien), combustion, } \\
\text { tours de refroidissement. }\end{array}$ & $\begin{array}{l}\text { Gouttelettes de nuages, } \\
\text { précipitation. }\end{array}$ \\
\hline Aérosols & $\begin{array}{l}\text { Combustibles fossiles et combustion de } \\
\text { biomasse, suie, activité volcanique, } \\
\text { poussière du sol, sel marin, plantes. }\end{array}$ & $\begin{array}{l}\text { Réduction due à la } \\
\text { précipitation. }\end{array}$ \\
\hline
\end{tabular}

Tableau 2: Sources et absorption de gaz à effet de serre et aérosols. Source : McMichel, d'après McGregor et Nieuwolt, 1998. Traduction et organisation: F. Mendonça.

Se basant sur l'analyse d'éruptions volcaniques, Molion (1994) insiste sur la thèse qu'il se produirait un refroidissement et non un réchauffement de la planète ; toutefois, c'est là une perspective contraire à la conviction de la plupart des chercheurs du monde entier. McGregor et Nieuwolt (1998, p.311) expliquent, à ce sujet, que l'impact des éruptions volcaniques varie spatialement (Amérique du Nord et Nord-Ouest du Pacifique) et temporellement (jours, mois ou années qui suivent l'éruption), vu que les réductions de température sont très peu ou pas du tout représentatives dans le bilan du réchauffement planétaire. On parle aussi de l’ODP (Oscillation Décennale du Pacifique) qui, dans un premier temps, aurait entraîné une élévation croissante des températures de l'atmosphère entre la fin des années 1940 et la fin des années 1990. Depuis 1997/1998, on serait d'ailleurs entré dans une période de refroidissement qui durerait jusqu’a la fin des années 2020. Cependant, bien que des réductions ponctuelles aient déjà été enregistrées, la tendance générale d’une élévation globale des températures de la planète se maintient.

Les débats les plus récents relatifs à la question du réchauffement global des deux derniers siècles défendent tous, de manière générale, la thèse que l'intensification de l'effet de serre planétaire serait directement liée aux modèles de production et de consommation de la société moderne l'augmentation de la concentration en GES dans la haute (basse?) troposphère provenant presque entièrement des activités humaines (industrie et agriculture, en particulier), Cette altération de la composition chimique atmosphérique aurait pour résultat 
un réchauffement de l'air et les prévisions les plus alarmantes annoncent, aux environs de l'an 2100 , une élévation de la température moyenne de la planète de l'ordre de $3,5^{\circ}$ à $6^{\circ} \mathrm{Cau}$-dessus de la température moyenne à l'heure actuelle, qui est de $16,5^{\circ} \mathrm{C}$ environ (IPCC, 2004).

Aussi bien l'intensité que la différenciation temporelle et spatiale du phénomène représentent encore de véritables enjeux pour les scientifiques de l'époque actuelle. Même si l'on croit que les changements les plus expressifs se produiront dans les moyennes et les hautes latitudes, les régions tropicales et équatoriales seront également soumises à des impacts climatiques non négligeables.

La création de futurs scénarios concernant les changements climatiques appartient encore au domaine de la spéculation, d'autant plus qu'il est difficile de comprendre de manière complète et satisfaisante le dynamisme de l'atmosphère (Monteiro, 1991). Il est aussi quasiment impossible de prévoir l'évolution des activités humaines, sujettes à des facteurs politiques, culturels, économiques et même à des facteurs liés à l'intervention de la nature en ce qui concerne la réalisation de ces activités. McGregor et Nieuwolt (op.cit. pg.303) considèrent, à ce propos, que le réchauffement le plus important se déroulera dans les plus hautes latitudes du fait de la réduction de la banquise. En même temps, ils prévoient qu'il y aura une intensification du cycle hydrologique et, par conséquent, une augmentation des précipitations, même si, sur ce dernier, point les scénarios climatiques restent plus imprécis.

Naturel ou anthropogénique, ou naturel et anthropogénique, le réchauffement de la troposphère est un fait du moment présent et de l'avenir proche et il faut agir de toute urgence pour le contrôler. Le problème a déjà éveillé l'attention de toute la société de la planète et sa dimension politique constitue l'un des points de discussion les plus conflictuels comme en témoignent les mouvements sociaux de la dernière décennie.

\section{Répercussions positives et négatives des changements climatiques}

Les changements climatiques globaux provoqueront des dispositions spatiales nouvelles et différenciées sur la surface de la planète et dans la vie des hommes. Tout en appartenant encore au domaine de la spéculation, les influences du réchauffement planétaire sur les conditions de santé et de maladie de la population doivent être prises au sérieux, car elles constituent une menace pour l'humanité. D'après Czeresnia et Ribeiro (2000, p. 12) «les conséquences épidémiologiques de cet intense processus de transformations sont radicales et imprévisibles. L'émergence de nouvelles maladies, qui peuvent aussi se manifester, sous forme d'épidémies fatales et dévastatrices, n'est pas seulement une fiction ».

Des répercussions positives et des répercussions négatives de l'intensification du réchauffement atmosphérique sont pronostiquées par 
différents spécialistes, quoique les répercussions négatives soient bien plus expressives et bien plus inquiétantes que les répercussions positives.

L'élévation de la fertilité des végétaux provenant de la plus grande fixation de carbone par les plantes et dans le sol en raison de l'augmentation de $\mathrm{CO} 2$ dans l'atmosphère, l'expansion des surfaces cultivables de la planète, particulièrement aux moyennes et hautes latitudes, et l'humidification des régions actuellement semi-arides (loin d'être encore observé!) en raison de l'élévation de la pluviométrie aussi bien que de l'hygrométrie, tous ces facteurs figureraient parmi les principaux effets positifs des changements climatiques.

Toutefois, la liste des répercussions négatives est bien plus longue que celle des répercussions positives. L'une des plus inquiétantes concerne l'augmentation du volume des eaux des océans (élévation de la température de la mer entre 1,4 à 5, $8^{\circ} \mathrm{C}$ entre 1990 et 2100) qui, s'associant au dégel partiel des glaciers et des calottes glaciaires des régions polaires, aurait pour résultat la hausse du niveau des mers de 0,4 à $1,5 \mathrm{~m}$, ce qui impliquerait la migration et la réinstallation dans d'autres régions d'une grande partie de l'humanité qui vit actuellement dans les régions côtières (Leggett, 1992).

Contrairement à l'expansion de la zone cultivable de la planète vers les zones tempérées, qui aura pour résultat une augmentation de la production agricole, la réduction des surfaces cultivables et de la production affecteront les zones tropicales et équatoriales actuelles. D’après Mendonça et al (2001, p. 105), la plus grande partie de la population de cette zone du globe souffre de la faim, elle est quasiment exclue du marché mondial et ses conditions de vie ne semblent pas s'améliorer avec l'intensification du réchauffement global.

Des situations de conflit entre les peuples et les nations pourront se déclencher par suite de problèmes liés à la faim, à la sécheresse et à la disponibilité de ressources. Ces nouvelles conditions pourront entraîner le déplacement et la migration forcée de la population; dans de pareilles situations, la société se voit confrontée à une gamme complexe de problèmes d'intensités diverses, mais présentant des résultats qui nuisent à la plupart de ses composants.

On constate, néanmoins, la simultanéité de l'accroissement de la population et de l'intensification du réchauffement global ; on observe, de manière assez claire, dans un tel contexte, que les effets des changements climatiques se répercuteront sur un nombre de plus en plus élevé d’hommes, car leur multiplication est plus rapide dans les groupes au revenu plus bas. Ce sont justement eux qui sont les plus assujettis aux impacts du «temps qui est lent et du temps qu'il fait » (Serres, 1989) ; c'est parmi eux que se trouvent ceux qui sont les plus atteints par les événements climatiques extrêmes. 
La répercussion des changements climatiques sur les conditions de santé et de maladie de la population exige une analyse toute spéciale à laquelle le présent texte va maintenant se consacrer.

\section{Réchauffement atmosphérique et maladies de la population}

Les études de géographie médicale, particulièrement la branche de la climatologie et le «complexe pathogénique » (Sorre, 1984), considèrent, de manière générale, l'étude du climat dans une perspective rétrospective pour comprendre après coup le présent et pour travailler ainsi avec les maladies transmissibles et parasitaires et avec celles qui dérivent directement des influences du climat sur les organismes vivants.

Ce n'est qu'actuellement, alors que les questions générales à propos des changements climatiques globaux et de leurs répercussions sur les conditions de vie aux échelles régionale et locale ont éveillé l'attention d'experts et de politiques, que l'examen attentif de leurs répercussions présentes et futures sur l'état de santé et sur les maladies des populations prend de l'importance (IPCC, 2004). Des épidémies inquiétantes de maladies émergentes, ré-émergentes et/ou récidivantes qui se sont déclarées pendant les deux dernières décennies dans diverses parties du monde ont fait l'objet principal de la reprise des études sur l'interaction du climat et de la santé des hommes (Mendonça, 2001, Mendonça et al, 2004).

Gatrel (2002,. p. 11-12) considère que les effets du réchauffement global sur la santé se feront sentir à long terme, alors que les effets provenant d'épisodes climatiques extrêmes se font sentir à court et très court terme. Dans ce dernier cas, il faut noter qu'il y a une interaction directe des impacts de phénomènes d'ordre naturel et des conditions sociales-économiques-technologiques des diverses sociétés humaines ; les sociétés moins bien loties sont plus exposées aux risques et, de ce fait, plus vulnérables que celles des pays riches et développés.

Tout en considérant que les zones tropicale et équatoriale seront celles qui subiront des impacts moindres en raison de l'intensification du réchauffement planétaire, on peut craindre, néanmoins, l'intensification de nombreuses maladies endémiques de ce côté-ci de la planète; à cela s'ajoute la simultanéité de l'expansion des régions où se déclare un grand nombre des maladies tropicales connues et de l'expansion des régions les plus chaudes dans des altitudes et dans des latitudes plus hautes. Des vagues de chaleur et de froid très intenses pourront être accompagnées de l'élévation des taux de mortalité dans des cas de maladies cardiovasculaires, cérébrovasculaires et respiratoires, sans oublier les problèmes déjà connus des cataractes et de cancer cutané dus a l'intensification du rayonnement ultraviolet. 
Besancenot (2001) signale que la morbidité et la mortalité provoquées par le réchauffement climatique seraient liées à six facteurs différenciés, présentant tous des interactions les uns entre les autres (figure 1); ces facteurs sont :

- l'élévation du niveau des mers,

- les paroxysmes météorologiques,

- l'agression de la chaleur,

- les effets sur la reproduction,

- la pollution atmosphérique,

- la nutrition.

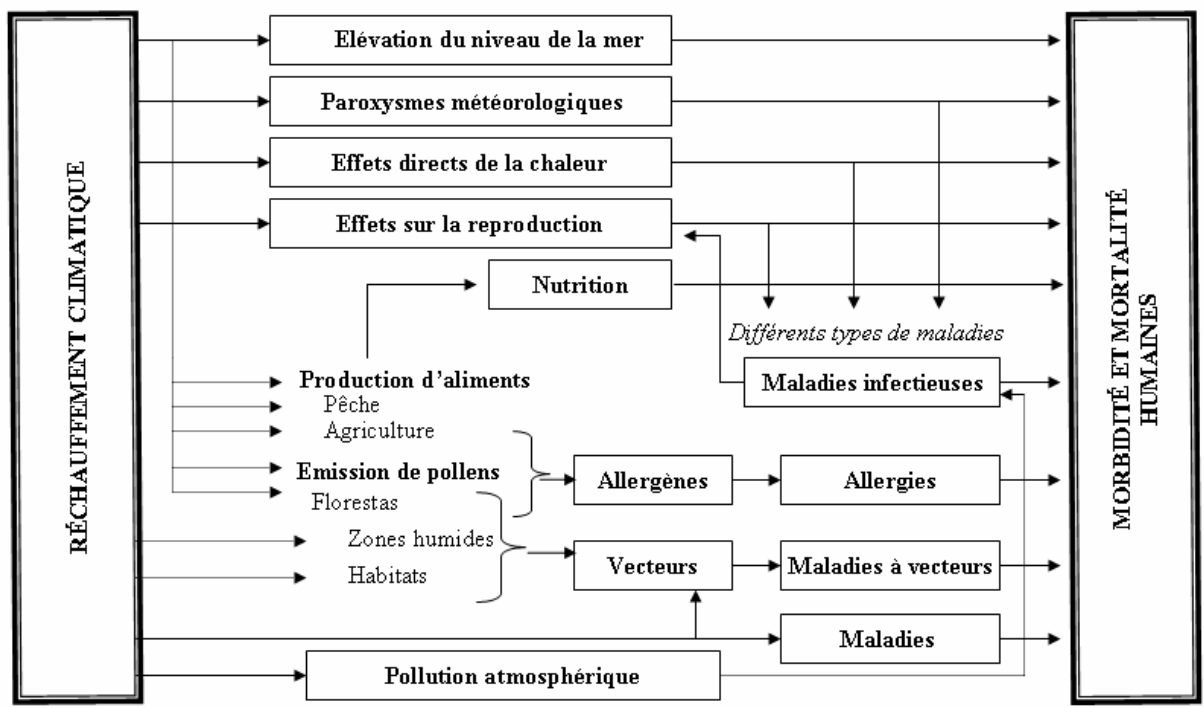

Figure 1 : Réchauffement climatique et santé, les interactions. Source : Besancenot, 2001. Global warming and health, interactions.

Les cinq premiers effets proviendraient directement du réchauffement climatique et le dernier en serait une conséquence indirecte, car il serait conditionné par les altérations subies au cours du processus de production d'aliments.

L'altération des forêts due au réchauffement global agirait sur les pollens et sur les allergènes, sur les zones humides, sur les habitats et sur les vecteurs, ce qui se répercuterait, respectivement, sur les allergies et sur les maladies transmises par des vecteurs.

L'augmentation de la chaleur serait favorable à l'expansion géographique des vecteurs de maladies transmissibles, alors que les maladies chroniques seraient directement affectées, élevant, de ce fait, les taux de morbidité et de mortalité de la population. Il se produirait une augmentation des cas de calculs rénaux, une élévation du taux de naissances prématurées et de mortalité 
périnatale, une multiplication des intoxications (en raison de la mauvaise conservation des aliments) ou encore un risque croissant de contamination des systèmes de climatisation et/ou d'humidification par des micro-organismes variés... "En fait, tout dépend de la brutalité avec laquelle s'opérerait le réchauffement » (Besancenot, 2001, p. 123).

En considérant les changements climatiques liés à l'effet de serre planétaire, Haines (1992, p.140) affirme que "différentes maladies, telles que le paludisme, la trypanosomiase, la leishmaniose, la filariose, l'amibiase, l'onchocercose, la schistosomiase et diverses maladies parasitaires vermineuses, aujourd'hui restreintes aux zones tropicales, sont liées à la température et pourraient théoriquement être affectées par le changement du climat». Les travaux de nombreux experts montrent que la température est aussi liée à beaucoup d'autres maladies non-parasitaires, telles que la fièvre jaune, la dengue et d'autres maladies virales transmises par des arthropodes, la peste bubonique, la dysenterie et d'autres affections diarrhéiques.

Dans une perspective à caractère de pronostic, Haines (1992), en émettant des hypothèses à propos des impacts du réchauffement global et du changement du climat sur la société, a aussi observé que ces impacts sont peut-être plus expressifs en ce qui concerne certaines catastrophes associées à des événements extrêmes, tels que les tempêtes et la famine. Il a considéré quelques problèmes liés à la santé mentale provenant de l'impact de la rupture de quelques barrages sur les survivants, pour projeter une image de situations futures telles que la hausse du niveau de la mer et le réchauffement de l'atmosphère pour ce qui est de leur influence sur la population.

Les interactions du réchauffement planétaire et des conditions de santé et de maladie de la population ont été invariablement conçues comme étant de deux natures en faisant la distinction entre les implications directes et les implications indirectes (Hufty, 1997; Besancenot, 2001 ; Gatrel, 2002). Ainsi ces implications peuvent être comprises, de manière générale, à partir de quelques exemples.

\subsection{Effets indirects sur les maladies en milieu tropical}

En considérant les effets indirects des changements climatiques, Gatrel (2002, p. 245) signale le fait qu'il ne faut pas manquer de concevoir la pollution comme l'un des facteurs associés à l'apparition de maladies déterminées ; il signale aussi l'intensification possible d'événements climatiques extrêmes, tels que les pluies très intenses, les tempêtes et les inondations, et des méfaits qu'ils entraînent. D’après Gatrel "des recherches suggèrent que les changements climatiques affecteront aussi bien les vecteurs que les agents infectieux qui transmettent les maladies infectieuses telles que le paludisme, la dengue et la trypanosomiase (maladie du sommeil)». 
En ce qui concerne le paludisme (vecteur : anophèle, parasite : plasmodium ou hématozoaire), les conditions environnementales qui favorisent son apparition sont une température de l'air située entre $20^{\circ}$ et $25^{\circ} \mathrm{C}$ (en dessous de cette température, le vecteur meurt), avec une pluviosité minimum de $1,5 \mathrm{~mm}$ par jour. L'action conjointe de la température et de l'humidité permet de contrôler le parasite : en Afrique, on a observé qu'au-dessus d'une température de $28^{\circ} \mathrm{C}$ il faut que la pluviosité soit de l'ordre de $80 \mathrm{~mm}$ environ pendant au moins cinq mois dans l'année pour permettre la reproduction du vecteur. (Gatrel, 2002, p. 72).

Considérant le réchauffement climatique de la planète, Gatrel (2002) émet l'hypothèse que, avec l'expansion des conditions typiques des régions tropicales, aires endémiques du vecteur, vers le Nord et vers le Sud (zone subtropicale de l'Europe, des Amériques, de l'Afrique, du Moyen-Orient et du Proche-Orient, du Sud de la Chine et de l'Australie) et en altitude (les Andes et la partie élevée de l'Afrique) le risque de transmission augmente; Martens affirme que l'anophèle se trouve déjà dans une grande partie de ces endroits mais que le parasite plasmodium, en revanche, ne peut pas survivre en raison des basses températures actuelles.

Des scénarios établis par certains chercheurs (Gatrel, 2002) signalent que la population globale en situation de risque potentiel sera comprise entre 2,4 et plus de 3 milliards de personnes et que de 220 à 480 millions d'entre elles, dont la plupart en Afrique, développeront la maladie. Ainsi, on observe que l'augmentation de l'apparition du paludisme en Asie est associée aux perturbations climatiques provoquées par le phénomène El Niño. Mais il faut aussi être attentif au fait que les vaccins et les nouvelles découvertes en médecine montrent que le climat n’est pas le seul facteur contraignant.

En étudiant l'apparition du paludisme aux environs du lac de l'usine hydroélectrique d'Itaipu, Costa Ferreira et Lombardo (1997) ont conclu qu'il y a eu une augmentation du nombre de cas enregistrés dans cette région ; toutefois, ils ont établi un rapport entre ce fait et le processus de migration de la population de régions endémiques au Brésil (Nord) vers cette région, un autre facteur étant l'intensification de l'humidité de l'air provenant de la rétention des eaux et de la formation du lac.

La dengue est une autre maladie qui subit indirectement les effets des changements climatiques; ses vecteurs sont les moustiques Aedes aegypti et Aedesalbopictus, le virus est de type Flavivirus. Les régions où elle se déclare communément sont l'Asie, l'Afrique, l'Amérique Centrale et l'Amérique du Sud, où on compte plus de 100 millions de cas par an. L'Organisation Mondiale de la Santé fournit des informations sur un type spécifique de dengue, la forme hémorragique, qui présente une très grande mortalité (environ 5\%) affectant principalement les enfants. "L'urbanisation rapide, les mouvements migratoires de la population, la résistance des moustiques aux insecticides et le 
stockage inadéquat d'eau propre sont les facteurs qui provoquent l'accroissement de l'apparition de la dengue » (Gatrel, 2002, p. 247). Pour ce qui concerne le Brésil, il est possible d'ajouter à ces aspects, les difficultés d'accès aux habitations, l'inefficacité du contrôle sanitaire (prolifération de zones de dépôts de pneus usages et de ferrailles, de terrains urbains non bâtis, de cimetières) et la résistance du vecteur aux larvicides et aux insecticides (Tauil, 2002).

La distribution des moustiques, leur longévité, la fréquence de leurs piqûres et la période d'incubation du virus sont affectées par la température ; à $27^{\circ} \mathrm{C}$, par exemple, la période d'incubation est de dix jours, à $34^{\circ} \mathrm{C}$, elle est de sept jours. Encore d'après Gatrel (2002), lors d'une élévation de l'ordre de $2^{\circ} \mathrm{C}$ de la température de la planète, la dengue se répandrait vers des régions telles que le Sud de l'Europe (Espagne et Grèce) et le Sud des États-Unis. De nos jours, les hautes latitudes sont libres de cette maladie en raison des basses températures, mais, de même que pour le paludisme, le changement climatique global pourra favoriser l'expansion de la dengue dans de nouvelles régions.

Des études très récentes relatives à la dengue ont mis en évidence son expansion géographique vers des régions qui ne sont pas considérées comme étant des régions endémiques de cette maladie ; comme preuve, on peut citer les exemples présentés pour le Nord de l'Argentine et pour la région de Buenos Aires (Bejaran et al., 2003), et pour les parties les plus élevées de l'État du Paraná au Brésil, en particulier la ville de Curitiba (située à une altitude moyenne de $900 \mathrm{~m}$ ) où on a enregistré les premiers cas autochtones de dengue en 2002 (Paula, 2002 ; Fernandes de Oliveira, 2003). L'augmentation des cas de dengue dans l'Etat du Parana s'accompagne d'une augmentation d'environ 1,2 C de la température moyenne ces trente dernières années; en 2003, année la plus chaude depuis les années 1980, on a enregistré plus de 9.000 cas, plutôt dans la partie nord de l'Etat (Mendonça et al., 2004).

La leptospirose est une zoonose d'importance reconnue dans la santé publique qui préoccupe de plus en plus les épidémiologistes et les médecins cliniques, tant à cause de la difficulté de son éradication, due à la complexité des aspects épidémiologiques auxquels la maladie est liée, qu’à cause du taux élevé de mortalité qu'elle provoque. La distribution géographique de la leptospirose est cosmopolite; malgré tout, les températures élevées et les saisons humides des tropicales et subtropicales favorisent l'occurrence de cette maladie et favorisent l'apparition de crise épidémique de nature saisonnière. En effet, le vecteur (le rat) et le virus (du genre Leptospire) de cette maladie dépendent de conditions de chaleur élevée et de fortes pluies pour que la transmission soit réalisée. De cette manière on constate que, avec l'augmentation de la température et des taux de pluviosité, ce qui est prévu pour ce siècle, la leptospirose pourra atteindre une population plus importante dans les zones tropicales du globe. L’intensification, en quantité et en qualité, des événements 
climatiques extrêmes tel qu'il a été constaté par l’IPCC (2004) ces vingt dernières années, parmi lesquels les inondations et les crues urbaines tropicales, met en évidence l'amélioration des conditions climatiques favorables au développement de cette maladie.

Intimement associée à la pauvreté, la leptospirose est particulièrement présente au Brésil. D'après le "GUIA BRASILEIRO DE VIGILANCIA EPIDEMIOLOGICA” de 1998 (données sur la période de 1985 à 1997), 35.403 cas de leptospirose humaine ont été enregistrés, variant de 1.594 cas annuels (minimum) en 1987 à 5.576 en 1997 (maximum), et présentant dans la même période une létalité moyenne de 12,5\%. En plus d'un processus d'intense urbanisation et de forte précarisation des conditions de vies urbaines au Brésil, comme dans tous les pays du Sud à cette époque, il faut noter que les températures et les pluies ont augmenté parallèlement. La recherche des corrélations entre ces variables est en cours, et il existe une forte probabilité que l'intensification de cette maladie tropicale soit reliée au réchauffement climatique global.

En ce qui concerne la maladie du sommeil (vecteur : la mouche tsé-tsé Glossina morsitans), dont la région endémique est constituée par les bordures du désert du Sahara en Afrique, l'élévation de la température de l'ordre de $1^{\circ} \mathrm{C}$ à $3^{\circ} \mathrm{C}$ pourrait également produire une expansion de la région où se déclare cette maladie.

L'intensification du réchauffement global pourra agir indirectement sur les intoxications alimentaires, elle influence, par exemple, la prolifération de la salmonelle en été, on pourrait citer d'autres exemples encore.

\subsection{Effets directs}

L'intensification de la concentration des GES dans la haute troposphère/basse atmosphère produit des altérations dans le bilan radiatif de la Terre. Dans ces conditions «certaines conséquences se feront sentir directement sur l'organisme humain, alors que d'autres se feront sentir à partir des conditions écologiques plus ou moins favorables à la survie, à la multiplication et au développement de tel ou tel germe pathogène, ou encore de tel ou tel insecte vecteur de ce germe " (Besancenot, 2001, p. 121).

Le stress thermique constitue un des principaux problèmes que la population va devoir affronter en raison du processus de réchauffement planétaire. McGregor et Nieuwolt (1998) affirment qu'il y aura une intensification de l'utilisation d'équipements de climatisation et que, malgré cela, chez les personnes âgées et les enfants, la morbidité et la mortalité présenteront des taux plus élevés qu'aujourd'hui à cause de l'augmentation des températures moyennes et maximales. 
Certains problèmes de santé provenant directement du réchauffement global et dont un nombre considérable d'experts pensent qu'ils se manifestent par le stress thermique peuvent être résumés comme suit :

- Diminution des décès l'hiver dans les hautes et moyennes latitudes et raréfaction des maladies de l'appareil respiratoire et des cardiopathies. En contrepartie, les décès augmenteront en été, ce qui est dû plutôt à l'augmentation des problèmes cardiovasculaires, cérébro-vasculaires, respiratoires, métaboliques, psychiques ou autres qu'à l'affaiblissement des mécanismes thermorégulateurs de l'organisme.

- En dessous du niveau de confort thermique, la mortalité augmente de $1 \%$ à chaque élévation de $1^{\circ} \mathrm{C}$ de la température de l'air ; au-dessus du niveau de confort thermique, elle augmente de $1,4 \%$ à chaque hausse de température de $1^{\circ} \mathrm{C}$.

- Dans le cas des maladies cardiovasculaires (pour les personnes âgées de plus de 65 ans), la mortalité baisse de $4 \%$ à une élévation de température de $1^{\circ} \mathrm{C}$ dans les pays à climat froid ; en régions tropicales, elle augmente de $1,6 \%$ à chaque degré.

- Pour ce qui est des maladies respiratoires, la mortalité baisse d'environ $4 \%$ et augmente d'environ $10,4 \%$ à chaque baisse ou hausse de température d'un degré, respectivement. Les enfants et les personnes âgées sont les personnes qui se trouvent dans des situations de plus grand risque, à cela s'ajoutent les conditions de pollution de l'air, quand il s'agit des maladies respiratoires et cardiovasculaires.

Gatrel (2002, p. 25) s’inquiétant à propos des répercussions planétaires des changements globaux, tout particulièrement de leurs répercussions sur la santé humaine, a présenté un modèle générique de l’impact du réchauffement global possible sur la santé. Il suggère, de manière générale, que, dans des endroits chauds

« comme Singapour, où le climat est chaud pendant toute l'année, la mortalité va augmenter, mais dans les villes au climat froid (Londres, par exemple) on observera de modestes élévations de la mortalité pendant les mois chauds, qui seront compensées par des réductions pendant les mois froids. La mortalité cardiovasculaire dans certains pays (pour les personnes âgées de plus de 65 ans) est mise en évidence dans le tableau $3^{1}$. Il subsiste encore bien des incertitudes en ce qui concerne ces scénarios ; ils dépendent de l'habileté des personnes à s'adapter physiologiquement aux changements de température. Cependant, la conclusion générale de Martens est que le réchauffement global réduira probablement la mortalité, particulièrement celle provoquée par des maladies cardiovasculaires, ce qui est dû au réchauffement de l'hiver. Des changements climatiques globaux réduiront, par conséquent, l'excès de mortalité en hiver, mortalité due aux bronchites, à la grippe et aux maladies cardiaques ».

\footnotetext{
${ }^{1}$ En accord avec ce chercheur
} 


\begin{tabular}{|l|c|c|}
\hline Pays & $\begin{array}{l}\text { Changement affectant la } \\
\text { mortalité liée au froid } \\
\text { (pour 100.000 personnes) }\end{array}$ & $\begin{array}{l}\text { Changement affectant la } \\
\text { mortalité liée à la chaleur } \\
\text { (pour 100.000 personnes) }\end{array}$ \\
\hline Singapour & 0 & 43 \\
\hline Japon & -79 & 18 \\
\hline Pays Bas & -181 & 19 \\
\hline Royaume Uni & -250 & 10 \\
\hline États-Unis & -184 & 32 \\
\hline Canada & -235 & 26 \\
\hline Espagne & -129 & 33 \\
\hline Australie & -98 & 22 \\
\hline
\end{tabular}

Tableau 3 : Estimation de changements du taux de mortalité cardiovasculaire en raison du stress thermique (Population d'un âge supérieur à 65 ans). Source : Martens, d'après Gatrel (2002, p. 245).

\subsection{Autres effets}

- Le nombre de personnes dans le monde qui vivent dans des régions sujettes à des inondations, nombre qui est proche de 100 millions, doublera pratiquement.

- Il se produira des impacts sur l'agriculture engendrant une réduction de la quantité de production d'aliments. Dans certaines régions, ces impacts auront pour résultat la mal-nutrition et la faim. D'après McGregor et Nieuwolt (1998, p. 308-309) «des changements climatiques dans les régions tropicales auront un nombre important de conséquences pour l'agriculture (...). (...). L'élévation des températures pourra aussi réduire le cycle de vie du maïs et du riz, entraînant une baisse de la production de ces céréales. Pour le Mexique, le Brésil et l'Uruguay, on peut prévoir un déclin de la production de maïs de 5 à $50 \%$ environ ».

- Des changements affectant l'usage du sol et l'augmentation des régions sujettes au déboisement, à la déforestation, etc. provoqueront l'altération des écosystèmes et des habitats naturels de certains vecteurs et, par conséquent, l'augmentation de l'apparition de certaines maladies, tout comme l'apparition de maladies infectieuses émergentes, telles que la fièvre hémorragique Ebola, par exemple.

- «(...) Des répercussions sur la santé sont aussi mises en rapport avec des migrations de la population et avec des changements dans la distribution des vecteurs de maladies » (Gatrel, 2002).

\section{Conclusion}

"Il faut bien le dire : l'action de l'environnement est indiscutable ; elle joue son rôle dans la formation des variétés physiques et mentales de l'humanité. Mais il faut admettre, en même temps, que son action 
n'est pas impérieuse, au point de déterminer, dans tous les cas, une spécialisation rigide. (...)».

Max. Sorre (1984, p. 71).

La reprise des études de géographie médicale et de la santé résulte de l'aggravation des conditions de vie de la plupart des êtres humains pendant cette fin du $\mathrm{XX}^{\mathrm{e}}$ siècle et au début de ce nouveau millénaire. Toutefois, bien que des progrès techniques et technologiques aient été réalisés afin de diminuer la souffrance des hommes quand ils sont atteints par des maladies, la concentration de ces maladies sur une petite partie de l'humanité montre que la plupart des êtres humains sont particulièrement vulnérables face aux risques encourus en cas d'accidents et de catastrophes naturelles. La notion de déterminisme environnemental doit toujours être réfutée, comme l'ont signalé Max Sorre (cf. l'épigraphe ci-dessus) et Mendonça (2001), entre autres, mais l'influence de la nature sur les conditions de la santé et de la maladie d'une population déterminée doit être revue dans une autre perspective, plus relativisée et interagissant avec d'autres facteurs du contexte culturel, politique et socioéconomique de la société:

«La manière dont le 'développement parcourt le sousdéveloppement' est la cause principale de la distribution inégale des problèmes de santé. Il soutient aussi bien la permanence des facteurs qui conditionnent l'environnement naturel que les profondes inégalités et iniquités sociales qui déterminent la vie, y compris celle des pratiques d'intervention dans la santé ».

(Iniguez Rojas, 1998, p.14)

Les changements climatiques globaux et leur manifestation la plus inquiétante, le réchauffement de la basse atmosphère, signalent aussi bien des altérations positives que des altérations négatives des paysages et des conditions de vie des hommes sur la surface de la planète. Les altérations négatives sont les plus importantes, car leurs répercussions peuvent faire redouter le déclenchement de conflits pour avoir accès à des ressources naturelles déterminées (sols, forêts, eau potable, etc.); de façon très spécifique, elles annoncent également l'expansion géographique et la recrudescence de certaines maladies, parmi lesquelles se distinguent les maladies transmissibles, parasitaires et infectieuses, dont l'apparition dépend directement des conditions climatiques.

Toutefois, il subsiste encore de considérables incertitudes à propos du réchauffement global, surtout quand on discute les causes de ce réchauffement. Dans un tel contexte, c'est la précaution qui semble être l'attitude la plus correcte pour la société ; pour ce faire, on peut mettre en oeuvre diverses initiatives afin de ralentir l'effet de serre planétaire, telles que la réduction du consumérisme et de la déforestation, par exemple. La stabilité de la température 
moyenne de la terre, ou la réduction des niveaux prévus, se répercutera sur la conservation de bonnes conditions de vie sur la planète, et même sur la création de nouvelles conditions de vie; des initiatives comme celles-ci ont tendance à se manifester directement dans les conditions de santé de la population.

Le réchauffement climatique global pourrait accentuer, à l'échelle du monde tropical, l'incidence du paludisme, de la dengue et de la leptospirose, parmi d'autres maladies fortement influencées par les conditions climatiques. Ces maladies sont, à l'heure actuelle, à la base d'une importante partie du taux de morbidité de la population de cette zone. Dans le cas du Brésil on a constaté durant les trente dernières années une intensification de l'incidence de ces maladies en même temps qu'une élévation de la température et de la pluviosité, surtout dans la partie sud du pays.

De toute façon, il semble évident que, plus on analyse la réalité, plus le réchauffement plus ou moins intense de la troposphère se répercutera de manière différenciée sur les hommes ; la manifestation évidente de l’inégalité des richesses et des accessibilités place la plupart des êtres humains dans des situations de pénurie. La réalité montre qu’il faut agir de toute urgence afin de freiner le réchauffement planétaire ; elle met aussi en évidence que cela ne se produira pas aussi longtemps que la concentration de la richesse et l'injustice sociale se maintiendra aux niveaux actuels. Si ces disparités persistent, les scénarios négatifs des changements globaux pourront avoir plus d'impact et réserver plus de surprises que ne le pensent les prévisionnistes.

\section{Bibliographie}

BEJARAN R. et al., 2002: Aplicacion de la predicción meteorológica para el pronostico de la abundancia potencial del Aedes aegypti en Buenos Aires, Revista Terra Livre, n. 20, São Paulo, Brasil: AGB., pp. 171-178.

BESANCENOT J-P., 2001 : Climat et santé, Paris, PUF. (coll. Médecine et société), $128 \mathrm{p}$.

CONFALONIERI U., 2001 : Global environnemental change and health. in: HOGAN D., TOLMASQUIM M. T. : Human dimension of global environmental change Brazilian perspectives, Rio de Janeiro, Academia Brasileira de Ciências. (p.79-97).

COSTA FERREIRA M. E. e LOMBARDO M. A., 1997 : A questão climática e a ocorrência de malária na área de influência do reservatório de Itaipu - PR - Brasil. Boletim Climatológico, ano 2, n. 3, FCT/UNESP, Campus de Presidente Prudente, julho. (p. 187-193).

CZERESNIA D., RIBEIRO A. M. 2000 : O conceito de espaço em epidemiologia: uma interpretação histórica e epistemológica, Cadernos de Saúde Pública, vol.16, $\mathrm{n}^{\circ} 3$, p.595-605.

FERNANDES DE OLIVEIRA M. M. 2003 : A dengue em Curitiba: Uma abordagem climatológica do episódio de março/abril-2002, Curitiba: UFPR, Monografia de conclusão de curso de graduação em geografia. Inédito. 
GATREL A. C. 2002 : Geographies of health, An introduction, Oxford, Blackwell Publishers Ltd.

GUIA BRASILEIRO DE VIGILÂNCIA EPIDEMIOLÓGICA, 1998 : 5 ed. Brasília : Fundação Nacional de Saúde.

HAINES A. 1992 : Implicações para a saúde, in: LEGGET, J. (Editor responsável). Aquecimento global - O relatório do Greenpeace. Rio de Janeiro: Editora da Fundação Getúlio Vargas, p. 135-148.

HUFTY, A. 1997 : Les climats urbains, in DUBREUIL V. et MARCHAND J.P. (ed.). Le climat, l'eau et les hommes - Ouvrage en l'honneur de Jean Mounier, Presses Universitaires de Rennes, p. 113-128.

INIGUEZ ROJAS L. 1998 : Geography and health: themes and perspectives in Latin America, Cadernos de Saúde Pública, out./dez., vol. 14, n 4, p. 701-711).

IPCC - InterGovernmental Panel on Climate Change. 2004 :

www.ipcc.ch/officialdocuments

LEGGETT, J. (Editor responsável), 1992: Aquecimento global - O relatório do Greenpeace, Rio de Janeiro, Editora da Fundação Getúlio Vargas, 516 p.

MCGREGOR G. R. and NIEUWOLT S. 1998 : Tropical climatology - An introduction to the climates of the low latitudes, Chichester, England, John Wiley and Sons, second edition, $339 \mathrm{p}$.

MENDONÇA F., 2000 : Aspectos da interação clima - ambiente - saúde humana : Da relação sociedade-natureza à (in)sustentabilidade ambiental, in RA'E GA - $O$ espaço geográfico em análise, v. 4, n 4, p.85-100.

MENDONÇA F., 2001 : Clima e criminalidade: Ensaio analítico da correlação entre a temperatura do ar e a criminalidade urbana, Curitiba: Editora da UFPR, 182 p.

MENDONÇA F. e NOGAROLI M., 2000; O Paraná no aquecimento global: Uma explicitação das relações geográficas entre o local e o global, in: FRESCA, T. M. et al. (Org.) : Dimensões do espaço paranaense, Série Geografia em Movimento 2, a editora é a UEL, as organizadoras são, Londrina, Editora da UEL. p. 129-152.

MENDONÇA F. et al., 2001 : A intensificação do efeito estufa planetário e a posição dos países no cenário internacional, in RA’E GA - O espaço geográfico em análise, $\mathrm{n}^{\circ}$ 5, ano V, Curitiba/PR, Editora da UFPR. p. 99-124.

MENDONÇA F. et al., 2004. : Réchauffement climatique global et expansion géographique de la dengue dans le Sud du Brésil, Actes du XVII Colloque de l'Association Internationale de Climatologie, Caen, France, p. 209-212.

MOLION L. C. B., 1994 : Efeitos de vulcões no clima, Caderno de Geociências, n 12 - out-dez, p 13-24.

MONTEIRO C. A. F., 1991 : Clima e excepcionalismo - Conjecturas sobre o desempenho da atmosfera como fenômeno geográfico, Florianópolis, Editora da UFSC, 233 p.

PAULA E. V. P., 2003: Relatorio de Pesquisa. Curitiba/PR: UFPR/PRPPG/IC. (Inédito). 
ROUQUAIROL, M. Z., 1993 : Epidemiologia e saúde, Rio de Janeiro, Medsi.

SERRES M. 1989 : La philosophie et le climat, in Colloque de Lassay Pollution, atmosphère, ozone, effet de serre, déforestation..., Paris, Larousse, p.50-61.

SORRE, M. 1984 : A adaptação ao meio climático e bio-social - geografia psicológica, in MEGALE, J. F. (Org.) : Max Sorre, São Paulo, Ática (Col. Grandes Cientistas Sociais, 46).

TAUIL P., 2002 : Aspectos críticos da dengue no Brasil, Cadernos de Saúde Publica, Rio de Janeiro, RJ; vol. 18, n³ 3 , p. 867-871.

WOODWELL G. M., 1992 : Os efeitos do aquecimento global, in LEGGET J. (Editor responsável) : Aquecimento global - O relatório do Greenpeace, Rio de Janeiro: Editora da Fundação Getulio Vargas. p. 106-120. 